\title{
PERCEPCIONES ACERCA DEL PROCESO DE BÚSQUEDA Y CONTRATACIÓN DE DOCENTES EN CHILE: NUDOS CRÍTICOS E INEQUIDAD DEL SISTEMA ${ }^{1}$
}

\author{
María Soledad Ortúzar \\ Pamela Ayala \\ Carolina Flores \\ Carolina Milesi ${ }^{2 *}$
}

\begin{abstract}
RESUMEN
Sobre la base de entrevistas en profundidad hechas a directores y jefes de UTP en 32 unidades de contratación de la Región Metropolitana de Chile, este estudio realiza una caracterización del proceso de contratación de docentes, abordando las prácticas y preferencias que se llevan a cabo en establecimientos de distinto nivel socioeconómico y dependencia. El análisis de las entrevistas mostró diferencias importantes en las percepciones de los equipos directivos de los establecimientos acerca de la complejidad y la formalidad del proceso de contratación. Los establecimientos privados subvencionados en redes grandes y con mayores recursos realizaban una gestión más compleja en el proceso de contratación. En todos los establecimientos del estudio se complementó lo que se describe en el currículum con al menos una entrevista presencial. Sin embargo, la observación de una clase demostrativa es menos común y se da en el caso de aquellos de mayores recursos. Deficiencias en los programas de formación inicial, salarios más bajos, condiciones de trabajo difíciles y falta de experiencia previa en contextos similares se señalaron como factores que dificultan la contratación de docentes calificados en establecimientos más vulnerables. En relación con los profesores nóveles, su adaptabilidad, vocación y disponibilidad a trabajar por salarios más bajos se mencionaron como ventajas para su contratación, en tanto que como desventajas se describieron el deficiente manejo de grupo, los problemas de autoridad y la falta de manejo de la diversidad en el aula. Los procesos de acompañamiento e inducción fueron percibidos como factores clave para la retención de docentes.
\end{abstract}

Palabras clave: contratación de docentes, distribución de docentes, establecimientos de bajo nivel socioeconómico, profesores recién egresados, retención de docentes.

1 Este trabajo contó con el financiamiento del Ministerio de Educación a través del Fondo de Investigación y Desarrollo en Educación (FONIDE N F711278).

2 Al momento de la realización de este estudio todas las autoras estaban afiliadas al Instituto de Sociología y al Centro de Políticas y Prácticas en Educación (CEPPE), ambos de la Pontificia Universidad Católica de Chile. Contacto: mortuzar@uc.cl. 


\title{
PERCEPTIONS ABOUT THE TEACHER HIRING PROCESS IN CHILE: CRITICAL ASPECTS AND INEQUITY IN THE SYSTEM
}

\begin{abstract}
Based on in-depth interviews with school leadership teams, compromised of principals and academic supervisors, from 32 Metropolitan Region recruitment units, this study characterizes the teacher recruitment process, studying practices and preferences in schools with different socioeconomic levels and governance. The analysis of the interviews shows important differences in the perceptions of school leadership teams on the complexity and formality of the recruitment process. Partially subsidized private schools, which belong to a large network and have greater financial resources, show a more complex management process in recruiting new teachers. In all of the schools studied, the resume is complemented by at least one in-person interview. Classroom observations are less common, however, and occur in higher-income schools. Weaknesses in initial teacher training programs, lower salaries, difficult job conditions, and a lack of previous experience in similar contexts are mentioned as factors explaining the failure to attract qualified candidates in low-income schools. Regarding new teachers, their adaptability, vocation and willingness to work for lower salaries are mentioned as advantages in their recruitment. Their disadvantages include group management, authority issues and a lack of diversity management in the classroom. Support and induction processes are perceived as key factors for teacher retention.
\end{abstract}

Keywords: teacher recruitment, teacher distribution, low-income schools, novice teachers, teacher retention.

\section{Introducción}

Abundante evidencia empírica describe a los docentes como el factor más importante para explicar el aprendizaje escolar (Barber \& Mourshed, 2007; Leithwood, Seashore, Anderson \& Wahlstrom, 2004; Sanders \& Rivers, 1996). Sin embargo, diversos estudios tanto en el contexto internacional (Akiba, LeTendre \& Scribner, 2007; Béteille \& Loeb, 2009; Loeb \& Reininger, 2004; Peske \& Haycock, 2006) como nacional (Cabezas, Gallego, Santelices y Zarhi, 2011; Ortúzar, Flores, Milesi y Cox, 2009; Paredes, Bogolasky, Cabezas, Rivero y Zahri, 2013; Rivero, 2015; Ruffinelli y Guerrero, 2009; Toledo y Valenzuela, 2012) revelan que los profesores se encuentran inequitativamente distribuidos, con un ordenamiento sistemático de profesionales menos calificados en establecimientos de menor nivel socioeconómico, espacios en los cuales hay precisamente una gran necesidad de profesores altamente calificados. Estos estudios se han centrado, en general, en factores asociados con las decisiones laborales 
de los docentes. Sin embargo, existe menos conocimiento acerca del posible rol que tienen las prácticas de contratación de los equipos directivos y sostenedores de los establecimientos educacionales en la distribución inequitativa de docentes en el sistema. Ante este vacío este estudio recoge de manera exploratoria la percepción de los distintos tipos de contratadores en relación con la búsqueda y la contratación de los profesores en Chile.

En términos de políticas públicas diversos esfuerzos a nivel nacional se han centrado en atraer y retener a mejores candidatos a la profesión docente, con el objetivo final de mejorar el aprendizaje de los alumnos y la calidad de la educación. Sin embargo, estas iniciativas no se han enfocado de manera suficiente en atraer a docentes altamente calificados a establecimientos más vulnerables, un aspecto fundamental si también se quiere lograr una mayor equidad educacional.

Dicho lo anterior, el objetivo general del presente trabajo es caracterizar el proceso de búsqueda y contratación de docentes, distinguiendo las tendencias y preferencias en los distintos tipos de contratadores y examinando particularmente las oportunidades de contratación para profesores con escasa o nula experiencia previa (o nóveles). Específicamente, se busca caracterizar las prácticas y preferencias tras el proceso y decisión de contratación desde la perspectiva de establecimientos educacionales de distinto nivel socioeconómico y dependencia, indagar en los nudos críticos de este proceso y describir las características asociadas al proceso de contratación de profesores nóveles y a la evaluación de su desempeño.

\section{Marco conceptual}

En esta sección se presenta evidencia respecto del proceso de contratación de profesores, tanto a nivel nacional como internacional. Se analiza el marco regulatorio, y los procesos de difusión y selección en la contratación de docentes, así como aspectos más específicos relacionados con la contratación de profesores nóveles y estrategias de retención de docentes. La evidencia respecto de los procesos de contratación es escasa tanto a nivel nacional como internacional $y$, en el caso de este estudio, la evidencia internacional se centra 
254 PERCEPCIONES ACERCA DEL PROCESO DE BÚSQUEDA Y CONTRATACIÓN DE DOCENTES EN CHILE: NUDOS CRÍTICOS E INEQUIDAD DEL SISTEMA - M. Ortúzar, P. Ayala, C. Flores, C. Milesi

mayoritariamente en Estados Unidos, por existir en dicho país mayor investigación respecto del tema.

\subsection{Marco regulatorio en la contratación de docentes}

Los requisitos para postular a cargos docentes varían según la dependencia del establecimiento educacional. En el sector municipal, el postulante debe cumplir con una serie de requerimientos estipulados por el Estatuto Docente 2011 (Art. 24). En cambio, para el sector privado -con o sin subvención del Estado-, los requisitos son definidos por cada empleador. Una situación similar ocurre en Estados Unidos: Dale Ballou y Michael Podgursky (1998) reportan que existen diferencias en los requisitos para postular a cargos docentes entre el sector público y privado, lo cual se traduce en que los establecimientos públicos tienen en general procesos más rígidos, mientras que el sector privado cuenta con mayor discrecionalidad. Si bien no existe evidencia respecto de cómo estas diferencias en los requisitos de contratación afectan las decisiones de empleo de los docentes, sí da cuenta de procesos de contratación distintos en ambos sectores.

Además de aspectos relacionados con la dependencia del establecimiento educacional, en Chile existe evidencia que revela que no todos los docentes disponen de las mismas oportunidades para postular a las ofertas laborales, debido a que tanto en el sector público como en el privado existen barreras formales e informales (distinciones culturales, familiaridad y contexto social del postulante) en el acceso a las ofertas. Así, el mercado laboral docente chileno se puede caracterizar como cuasisegmentado, lo cual implica que los profesores no siempre postulan a los colegios con mejores condiciones laborales y mejores salarios, dado que puede haber uno o más segmentos que están ausentes en su proceso de búsqueda. Los investigadores señalan, además, que existe poca evidencia respecto de cuáles son dichas barreras y cómo operan, y sugieren mayor investigación para identificarlas con el fin de ir eliminándolas a lo largo del tiempo (Paredes et al., 2013). 


\subsection{Difusión de la oferta laboral docente}

Los procesos de difusión de las ofertas laborales podrían tener una incidencia en la calidad de los maestros contratados, dado que independientemente de las preferencias y autoselección de los potenciales postulantes, estrategias de difusión efectivas permiten llegar al público deseado con información acerca de la oferta laboral (Balter \& Duncombe, 2005).

En el caso chileno, la difusión de la oferta laboral es definida de modo más formal en el sector municipal. En este, el proceso se verifica mediante concursos públicos de carácter nacional, que se efectúan al menos una vez al año, dentro de plazos establecidos.

No obstante, Grau, Maturana, Paterson y Valenzuela (2012) observan que, a pesar de que los sectores municipal, particular subvencionado y particular pagado poseen estructuras internas disímiles, no existen mayores diferencias en términos de difusión de las ofertas laborales.

Los autores señalan que los medios más utilizados para hacerlo son el diario, los sitios web de portales laborales y el contacto de quienes realizaron su práctica laboral en el establecimiento. En esta etapa son altamente valorados los contactos personales de los mismos profesionales del establecimiento, dado que es una forma de acceder a referencias directas acerca de los postulantes (Grau et al., 2012). Estos contactos son especialmente relevantes para la búsqueda de reemplazos, donde el proceso de contratación suele ser más flexible, dado el carácter de "imprevisto" de la situación (Paredes et al., 2013).

\subsection{Técnicas de selección de postulantes}

En cuanto al método de selección de docentes en Chile, nuevamente se observan diferencias según la dependencia del establecimiento. Es el sector municipal el que tiene una estructura más definida en términos formales. En este, el proceso de selección es llevado a cabo por una 
Comisión Calificadora de Concurso ${ }^{3}$. Dada la estructura centralizada de este proceso, los establecimientos municipales pueden ver limitadas sus posibilidades de seleccionar profesores de acuerdo con sus necesidades, ya que tienen poca participación en el proceso y en la comisión que finalmente decide. No así en los establecimientos con sostenedores privados, donde no existe un método de selección establecido por la ley ni estandarizado, por lo que cada escuela define sus propios parámetros de selección. En este aspecto un estudio realizado por Corvalán, Elacqua y Salazar señala que en escuelas municipales, tanto directores como jefes de UTP manifiestan tener poca influencia en la selección de profesores, mientras que en el caso de escuelas subvencionadas y particulares privadas existe mayor autonomía (Corvalán et al., 2009).

En una primera etapa del proceso de selección de docentes, las técnicas más comúnmente utilizadas son la revisión de currículum vitae (CV) por parte de directores, equipos directivos y, en algunos casos, sostenedores (Paredes et al., 2013). A su vez, los establecimientos suelen solicitar otros documentos, como el certificado de título, papel de antecedentes y referencias. En este proceso habitualmente quedan fuera instrumentos evaluativos relevantes (como evaluación docente, puntaje en prueba INICIA o nota de egreso), los que podrían dar luces del desempeño del candidato (Grau et al., 2012).

La cantidad y el tipo de instrumentos utilizados para seleccionar a los docentes también pueden variar según el nivel de recursos de los establecimientos. La evidencia es limitada en este aspecto, sin embargo, Balter y Duncombe (2005) en su estudio referido a prácticas de contratación de profesores en el estado de Nueva York señalan que en los distritos con menores recursos se suelen solicitar en menor medida antecedentes que otorgan información acerca de las habilidades docentes (como la especialización en la materia a impartir, portafolio, rendimiento), herramientas que sí se solicitan de manera más frecuente en aquellos de mayores recursos.

3 Estas comisiones están integradas por el director del Departamento de Administración de Educación Municipal de la Corporación Municipal que corresponda, o a quien se designe en su reemplazo; el director del establecimiento que corresponda a la vacante concursable; un docente elegido por sorteo entre los pares de la especialidad de la vacante a llenar; y el secretario municipal de la respectiva comuna, quien actúa como ministro de fe. 
Una segunda etapa en el proceso de selección consiste en la ronda de entrevistas. Quien la realiza suele ser el director, acompañado, en algunos casos, por el jefe de UTP, psicólogo, sostenedor o administrativos del municipio (en el caso de la educación pública chilena). Una práctica menos frecuente es la realización de una clase expositiva y la rendición de una prueba de conocimiento disciplinar, las que tienden a darse más bien en el sector particular (Grau et al., 2012).

Un fenómeno relevante dentro del proceso de contratación refiere a las interacciones entre los postulantes y la escuela. Cuando se da este intercambio de información -por ejemplo, por medio de entrevistas con un amplio sector de la comunidad escolar, clases demostrativas, observación de clases del staff de la escuela o reuniones con el personal-, tanto el candidato como quien realiza la contratación tienen la oportunidad de recopilar información y formar impresiones respecto del otro. Por el contrario, los procesos pobres en información, utilizan actividades que proporcionan datos insuficientes o que van en una sola dirección (desde el postulante al director). Los procesos ricos en traspaso de información tienen como consecuencia una menor tasa de rotación y los docentes declaran sentirse más satisfechos con su trabajo, debido a que recibieron una imagen clara y precisa del establecimiento (Liu, 2005).

Por otra parte, las características personales y académicas que buscan los establecimientos al momento de realizar una contratación también ejercen un rol importante en el proceso de selección. Algunas investigaciones realizadas en Estados Unidos señalan que los empleadores suelen poner atención en el desempeño durante las entrevistas personales y en las credenciales universitarias, más que en otros elementos, como podría ser la observación de una clase (Boyd, Lankford, Goldhaber \& Wyckoff, 2007; Boyd, Lankford, Loeb \& Wyckoff, 2013). Al mismo tiempo, existe el caso de directores que reconocen no reparar demasiado en el currículum académico del postulante, ya que existe la idea de que una persona "mejor capacitada" en términos académicos puede tener otro tipo de falencias a nivel personal, y que además existe el riesgo de renuncia en búsqueda de mejores oportunidades. Es así que para la selección 
se busca una mezcla entre raza, género y experiencia, ya que se considera que todos los alumnos debieran tener profesores a los que puedan ver como modelos a seguir (Harris, Rutledge, Ingle \& Thompson, 2010).

\subsection{Profesores nóveles y retención de docentes}

La retención de los docentes durante los primeros años de ejercicio de su carrera profesional ha cobrado creciente interés. El análisis de la retención docente tiene dos niveles; el primero se relaciona con la retención dentro del sistema educativo, mientras que el segundo con la retención dentro de las escuelas. En el último caso, el análisis se plantea como la retención de "buenos" docentes dentro de los establecimientos.

Respecto de la retención dentro del sistema educativo, la evidencia chilena destaca que un porcentaje considerable de docentes se retira de la profesión durante los primeros años de su trayectoria laboral -un 23\% abandona al segundo año, y un 40\% al quinto año-, lo cual se explica por las precarias condiciones laborales y el débil liderazgo escolar (Centro de Investigación Avanzada en Educación, CIAE, 2014). Esto no es exclusivo para Chile: en Estados Unidos el $40 \%$ de los docentes se retira antes de comenzar el primer año de ejercicio, mientras que en el Reino Unido, el 40\% de los docentes dejan la profesión en los primeros tres años de trabajo en la docencia (Cooper \& Alvarado, 2006). En relación con la retención dentro de las escuelas nuevamente existe una situación de inequidad: la retención de profesores más calificados en establecimientos de menor nivel socioeconómico es sistemáticamente menor que en establecimientos de mayores recursos (Darling-Hammond, 2003; Ingersoll, 2001).

Uno de los elementos más recurrentes en la discusión sobre la retención de los docentes son los incentivos salariales. Sin embargo, junto con un buen salario, las mayores tasas de retención se asocian a mejores condiciones laborales en general, como lo son políticas de inducción de calidad y un mayor apoyo del equipo directivo de los establecimientos (Guarino, Santibáñez, Daley \& Brewer, 2004; Johnson, 2006; Ladd, 2011; Loeb, Darling-Hammond \& Luczak, 2005; Weiss, 1999). 
En este ámbito, existen investigaciones que plantean que las políticas de retención basadas en incentivos salariales, además de ser costosas, resultan ineficientes, mientras que las basadas en condiciones de trabajo -como contar con el apoyo del equipo directivo y apoderados- terminan siendo mejores medidas (Hanushek $\&$ Rivkin, 2007). De modo adicional al apoyo, Futernick (2012) destaca que para la retención de los docentes inciden la asignación adecuada de cursos -es decir, que se designen las asignaturas de acuerdo con las credenciales de los docentes-, contar con un ambiente seguro, el acceso apropiado a los materiales de enseñanza, y que el tamaño de los cursos sea manejable. Este último aspecto se reafirma a la luz de los datos proporcionados por Guarino et al. (2004), donde a medida que crece la proporción de alumnos por aula, la retención de docentes es menor.

En Chile un aspecto importante de considerar al analizar la retención docente se relaciona con la formación inicial. Algunos estudios muestran que los programas de formación inicial en el país no han alcanzado niveles óptimos de calidad y, por lo tanto, los docentes no se encontrarían bien preparados para insertarse dentro de la profesión (Ávalos, 2014; Ruffinelli, 2013). Esto cobra mayor relevancia cuando los profesores se insertan en contextos vulnerables, ya que hay estudios que muestran que las principales debilidades percibidas por estos respecto de dichos programas se relacionan con la preparación para la diversidad y el manejo de la disciplina en la sala de clases (Gaete, Castro, Pino y Mansilla, 2016; Rufinelli, 2013), ambos aspectos muy relevantes al trabajar en contextos de pobreza.

A la luz del caso de Taiwán, en el que los profesores principiantes son proclives a mantenerse en la carrera docente, Wang \& Fwu (2013) proponen una serie de medidas para proporcionar el apoyo necesario durante los primeros años de ejercicio docente. Primero, sugieren la realización de mentorías que fortalezcan la colaboración entre la universidad y la escuela, ayudando a reducir la brecha entre la teoría y la práctica. Así mismo, los autores proponen el aumento de los salarios, la autonomía de las escuelas para incorporar prácticas efectivas e innovar y el aumento del prestigio de la profesión docente. 
A la luz de esta literatura nacional e internacional, a continuación se presenta nueva evidencia acerca del proceso de búsqueda y contratación docente, en el contexto chileno.

\section{Metodología}

La investigación se realizó a través de un estudio colectivo de casos, metodología que es definida por Stake $(1995 ; 2005)$ como el estudio de la particularidad y de la complejidad de un conjunto de casos, los que han sido elegidos bajo la premisa de que la comprensión de ellos llevará a un mejor entendimiento teórico, al ser más extensiva la recogida de información.

Dado el tiempo y los recursos disponibles, se trabajó solo en la Región Metropolitana, donde se analizó comprensivamente el proceso de búsqueda y contratación de profesores a partir del discurso de quienes participan de este proceso. Se analizaron casos correspondientes a distintos tipos de sostenedores con la finalidad de documentar una posible variación en el proceso de contratación entre establecimientos, sus preferencias y nudos críticos.

\subsection{Casos de estudio}

Dada la diversidad del sistema escolar chileno, se definieron seis tipos de sostenedores públicos y privados entre los cuales potencialmente existía variación en el proceso de contratación. Dada la poca evidencia que existe en Chile respecto de este tipo de procesos, los criterios para la identificación de las unidades contratadoras se basan en las diferencias administrativas que existen dentro del sistema público y del sector privado, así como el grupo socioeconómico (GSE) del caso en cuestión.

Al interior de cada grupo fueron seleccionados, aleatoriamente, los casos de estudio, que en total suman 32. En la Tabla 1 se observa la distribución de los casos posibles y los casos seleccionados para cada tipo de sostenedor o unidad contratadora definida en el marco de esta investigación. 
Tabla 1. Casos posibles y casos seleccionados para el estudio en la Región Metropolitana

\begin{tabular}{|c|c|c|c|c|}
\hline \multicolumn{3}{|l|}{ Tipo } & $\begin{array}{l}\text { Casos } \\
\text { posibles }\end{array}$ & $\begin{array}{c}\text { Casos } \\
\text { seleccionados }\end{array}$ \\
\hline \multicolumn{5}{|c|}{ Sector público o municipal* } \\
\hline & DAEM & & 23 & 3 \\
\hline & Corporaciones & & 29 & 3 \\
\hline \multicolumn{5}{|c|}{ Sector privado } \\
\hline & \multicolumn{2}{|c|}{ Privado subvencionado } & & \\
\hline & & Grandes redes** & 6 & 3 \\
\hline & & $\begin{array}{l}\text { Escuelas en red } \\
\text { descentralizadas }\end{array}$ & 195 & 8 \\
\hline & & Escuelas independientes & 686 & 11 \\
\hline & \multicolumn{2}{|c|}{ Privado no subvencionado } & & \\
\hline & & Escuelas privadas & 236 & 4 \\
\hline
\end{tabular}

Fuente: Elaboración propia a partir de Directorio de establecimientos 2012.

* Cantidad de municipios, los que en total reúnen 586 establecimientos en la RM.

** Cantidad de grandes redes, las que en total reúnen 47 establecimientos en la RM.

Para representar al sector público, se seleccionaron seis municipios de la RM (de un total de 52), tres de ellos gestionan la educación a partir de Departamento de Administración de Educación Municipal (DAEM) y tres lo hacen a partir de Corporaciones Municipales. Los seis municipios seleccionados tienen distinta concentración de pobreza escolar municipal ${ }^{4}$, la que fluctúa entre un $10 \%$ y un $100 \%$.

En cuanto al sector privado, se seleccionaron establecimientos particular subvencionados por el Estado y particulares no subvencionados (particulares pagados).

Dentro de los establecimientos particular subvencionados se distingue entre establecimientos independientes (sostenedor que administra solo una escuela) y establecimientos pertenecientes a redes educativas (sostenedor privado que administra más de una escuela). Sin embargo, dentro de este último grupo, existen diferencias en la forma en que se llevan a cabo los procesos de contratación. Según datos recolectados por este equipo a través de llamados telefónicos

4 Porcentaje de establecimientos públicos de nivel socioeconómico bajo o medio bajo por sobre el total de establecimientos públicos de la comuna. 
262 PERCEPCIONES ACERCA DEL PROCESO DE BÚSQUEDA Y CONTRATACIÓN DE DOCENTES EN CHILE: NUDOS CRÍTICOS E INEQUIDAD DEL SISTEMA - M. Ortúzar, P. Ayala, C. Flores, C. Milesi

realizados en enero 2013, de las 170 redes clasificadas como sociedades, fundaciones o congregaciones religiosas, un $68 \%$ contrata a los docentes de forma descentralizada, es decir, desde los mismos establecimientos educativos que conforman la red. El 32\% restante, realiza el proceso de contratación de forma centralizada.

Con base en ello, se seleccionaron 11 establecimientos privados subvencionados independientes y ocho establecimientos privados subvencionados que forman parte de una red, pero que presentan contratación independiente. Para representar a los establecimientos que pertenecen a redes que contratan de forma centralizada, se seleccionaron tres grandes redes educativas de la RM.

Finalmente, y dada la homogeneidad interna del grupo de establecimientos privados pagados, se seleccionaron aleatoriamente cuatro casos.

Adicionalmente se distinguió según el grupo socioeconómico del caso en cuestión (alto, medio y bajo), sobre la base de la clasificación que se realiza en el marco de la prueba Simce y los niveles de pobreza de la comuna seleccionada. En la Tabla 2 se expone la distribución de los casos según estos criterios de clasificación.

Tabla 2. Casos seleccionados para el estudio en la Región Metropolitana según nivel de pobreza escolar en el Municipio o GSE del establecimiento

\begin{tabular}{|c|c|c|}
\hline \multicolumn{2}{|l|}{ Tipo } & $\begin{array}{c}\text { Casos } \\
\text { seleccionados }\end{array}$ \\
\hline \multicolumn{3}{|c|}{ Sector público o municipal: nivel de pobreza escolar en el Municipio } \\
\hline & Mayor (más de 13,7\%) & 2 \\
\hline & Media (entre $9,6 \%$ y $13,7 \%$ ) & 2 \\
\hline & Menor (hasta $9,5 \%$ ) & 2 \\
\hline \multicolumn{3}{|c|}{$\begin{array}{l}\text { Sector privado (subvencionado y no subvencionado): GSE del establecimiento } \\
\text { según SIMCE } 2012\end{array}$} \\
\hline & Grandes redes & 3 \\
\hline & \multicolumn{2}{|l|}{ Establecimientos } \\
\hline & \begin{tabular}{|l|l|} 
& Grupo bajo $(A \circ B)$ \\
\end{tabular} & 8 \\
\hline & Grupo medio (C) & 5 \\
\hline & Grupo alto (D o E) & 10 \\
\hline
\end{tabular}

Fuente: Elaboración propia a partir de Directorio de establecimientos 2012, SINIM y bases de datos de establecimientos Simce 2012. 


\subsection{Instrumentos de recolección de datos}

El estudio de casos se desarrolló sobre la base de la aplicación de entrevistas a los equipos directivos de los establecimientos educativos, municipios y grandes redes educativas.

En una primera etapa, se realizaron entrevistas semiestructuradas a los equipos directivos involucrados en la contratación de profesores en los 32 casos de estudio, esto con el objeto de indagar en el proceso de llamado a concurso y de recolección de CV, conocer el proceso y los criterios de selección e identificar a los participantes en las decisiones de contratación.

En una segunda etapa, se realizaron entrevistas en profundidad a actores que participan del proceso de contratación y que, además, podían reportar información referida al desempeño de las nuevas contrataciones en el aula ${ }^{5}$. Así, en cada caso de estudio fueron entrevistados directores o jefes de UTP, privilegiando a estos últimos cuando se identificaron contrataciones a profesores principiantes o jóvenes.

Las entrevistas a directores tenían por objetivo evaluar la propensión a contratar cierto tipo de profesores por sobre otros e indagar acerca de los nudos críticos en el proceso de selección y las estrategias para superarlos. Por su parte, las entrevistas a jefes de UTP buscaron profundizar en la evaluación del desempeño de los profesores recién contratados, en particular de aquellos con poca o nula experiencia previa, en el proceso de adaptación a la realidad del establecimiento, y en la forma en que las escuelas apoyan a los profesores nuevos en el proceso de adaptación.

5 En cada municipio y red educativa fueron seleccionados dos establecimientos de forma aleatoria. Solo un municipio no participó de esta segunda etapa. 
264 PERCEPCIONES ACERCA DEL PROCESO DE BÚSQUEDA Y CONTRATACIÓN DE DOCENTES EN CHILE: NUDOS CRÍTICOS E INEQUIDAD DEL SISTEMA - M. Ortúzar, P. Ayala, C. Flores, C. Milesi

Tabla 3. Temas abordados en las entrevistas a directores y jefes de UTP

\begin{tabular}{|c|c|}
\hline \multicolumn{2}{|c|}{ Entrevistas a directores } \\
\hline Tema 1 & $\begin{array}{l}\text { Nudos críticos del proceso de contratación: } \\
\text { Razones tras la necesidad de iniciar un proceso de contratación. } \\
\text { Forma en que se realiza el llamado a concurso, fechas y razones por las que se hace en } \\
\text { determinada fecha. } \\
\text { Recolección de postulaciones (CV y otros formatos de postulación). } \\
\text { Proceso de selección (actividades realizadas y documentos solicitados). } \\
\text { Participantes del proceso de selección (rol y funciones en el proceso). }\end{array}$ \\
\hline Tema 2 & $\begin{array}{l}\text { Preferencias de directores a la hora de contratar nuevos profesores: } \\
\text { Estrategias seguidas para buscar profesores que cumplan con el perfil deseado. } \\
\text { Dificultades para encontrar a profesores que cumplan con el perfil deseado. } \\
\text { Estrategias para retener a profesores que cumplen con el perfil deseado dentro del } \\
\text { plantel. }\end{array}$ \\
\hline Tema 3 & $\begin{array}{l}\text { Preferencia por contratación de profesores jóvenes/mayores principiantes/con } \\
\text { experiencia (razones tras las preferencias). }\end{array}$ \\
\hline Tema 4 & $\begin{array}{l}\text { Solo en municipios y grandes redes: } \\
\text { Autonomía de las escuelas en el proceso de contratación y selección. } \\
\text { Forma en que participa el municipio o red en el proceso de selección. }\end{array}$ \\
\hline \multicolumn{2}{|r|}{ Entrevistas a jefes de UTP } \\
\hline Tema 1 & $\begin{array}{l}\text { Preferencias de jefes de UTP a la hora de contratar nuevos profesores: } \\
\text { Estrategias seguidas para buscar profesores que cumplan con el perfil deseado. } \\
\text { Dificultades para encontrar a profesores que cumplan con el perfil deseado. } \\
\text { Estrategias para retener a profesores que cumplen con el perfil deseado dentro del } \\
\text { plantel. }\end{array}$ \\
\hline Tema 2 & $\begin{array}{l}\text { Evaluación de los profesores jóvenes o principiantes: } \\
\text { Evaluación general del desempeño (conocimiento disciplinario y práctico, desempeño } \\
\text { en el aula, manejo de la disciplina y del clima dentro del aula, capacidad de evaluar } \\
\text { contenidos, de llegar a los alumnos con mayores dificultades y en la planificación). } \\
\text { Percepciones respecto de las fortalezas y debilidades en el desempeño. } \\
\text { Rol de la formación inicial docente en el desempeño de los profesores jóvenes o } \\
\text { principiantes. } \\
\text { Estrategias para enfrentar las dificultades en el desempeño de los profesores jóvenes o } \\
\text { principiantes. }\end{array}$ \\
\hline Tema 3 & $\begin{array}{l}\text { Proceso de adaptación al establecimiento: } \\
\text { Apoyo a los profesores nuevos en general y a los nuevos jóvenes y recién egresados. } \\
\text { Estrategias de inducción o mentoría/profesor guía. }\end{array}$ \\
\hline
\end{tabular}

Fuente: Elaboración propia a partir de pautas de entrevistas diseñadas para el estudio.

En total, se realizaron 33 entrevistas a directores y jefes de UTP $^{6}$. La distribución de los entrevistados según tipo de unidad de contratación se resume en la Tabla 4.

6 Cabe destacar que dos de las escuelas privadas seleccionadas en la primera etapa no accedieron a participar de las entrevistas en profundidad a directores y jefes de UTP. 
Tabla 4. Cantidad de entrevistas según unidad de contratación

\begin{tabular}{|c|c|c|c|c|}
\hline Tipo & & Director & Jefe de UTP & Total \\
\hline \multicolumn{2}{|c|}{ Sector público o municipal } & 5 & 5 & 10 \\
\hline \multicolumn{5}{|c|}{ Sector privado } \\
\hline \multicolumn{5}{|c|}{ Privado subvencionado } \\
\hline & Grandes redes & 3 & 3 & 6 \\
\hline & \begin{tabular}{|l} 
Escuelas en red \\
descentralizadas o \\
independientes
\end{tabular} & 9 & 6 & 15 \\
\hline \multicolumn{5}{|c|}{ Privado no subvencionado } \\
\hline & Escuelas privadas & 1 & 1 & 2 \\
\hline Total & & 18 & 15 & 33 \\
\hline
\end{tabular}

Fuente: Elaboración propia a partir de Directorio de establecimientos 2012, SINIM y bases de datos de establecimientos Simce 2012.

\subsection{Métodos de análisis de datos}

Los datos obtenidos a partir de las entrevistas en profundidad fueron examinados siguiendo una metodología cualitativa que buscó identificar y relacionar los principales conceptos y categorías del material levantado. A partir de los principios de la grounded theory (Glaser \& Straus, 1967), se enfrentó al contenido de manera inductiva, generando conceptos e ideas que emergen desde los datos, en contraste con conceptos que se desarrollan sobre la base de códigos preestablecidos y que después simplemente son testeados con los datos empíricos (Maxwell, 2013). De esta forma se generaron códigos referidos a diversos temas, que luego se agruparon en categorías temáticas, y así hasta llegar a un nivel de descripción suficientemente detallado y saturado en torno al fenómeno de la contratación de profesores en Chile.

En concreto, el análisis de las entrevistas se realizó mediante tres etapas. La primera consistió en una lectura general de todo el material, sin diferenciar entre los tipos de unidades contratantes de nuestros entrevistados. El objetivo de esta primera lectura fue generar, mediante codificación abierta, una lista de conceptos y temáticas transversales en las entrevistas, incluyendo discursos emergentes que no hubiesen sido abarcados en la pauta de entrevista.

Una vez identificados y registrados dichos conceptos, se procedió a ordenarlos y entender sus aristas que dieron forma 
a la estructura del informe. Para esto, se releyó cada entrevista detalladamente, esta vez buscando citas y contenidos específicamente relacionados con los conceptos levantados en la etapa primera. Como resultado de esta segunda ronda, se elaboró un nuevo documento de trabajo que reagrupó el texto de las entrevistas en un anexo de citas. En él se ordenaron los comentarios de cada entrevistado relacionados con cada concepto, con el fin de identificar diferencias y similitudes dentro de las fuentes respecto del mismo concepto, al igual que para encontrar relaciones y agrupaciones entre ellos. El análisis de relaciones permitió establecer un orden entre conceptos (categorías) que dieron pie al orden lógico del relato. Así, se optó por utilizar dichas categorías como subcapítulos, dentro de los cuales se relacionaban y describían distintos conceptos.

Una vez definido el orden temático, en una tercera etapa de análisis, se revisaron las diferentes posturas entre entrevistados según el tipo de establecimiento educativo que representan. También se releyó la lista de conceptos generada en la primera etapa, para revisar que todos estuvieran debidamente integrados en el texto de resultados.

Cabe destacar que este es un estudio exploratorio en el que conceptos clave como aquel de calidad docente se abordan desde la perspectiva de los contratadores, de modo que discursos y teorías que emergen en torno a estos se relacionan con las preferencias y percepciones de los actores en el proceso de contratación docente.

\section{Resultados}

A continuación se exponen los principales resultados del estudio, los que se dividen en dos grandes ítems. En primer lugar, se presentan los estándares generales de las decisiones de contratación. Se aborda la forma en que se realiza el llamado a concurso, cómo opera el proceso de selección, los criterios utilizados para determinar si un profesor es idóneo para el cargo, las dificultades en el proceso de contratación, la rotación y las estrategias llevadas a cabo para retener a los profesores. En segundo lugar, se profundiza en la contratación de profesores jóvenes o recién egresados. En este punto se destacan sus fortalezas y la evaluación que hacen los jefes de UTP del desempeño en el aula. 


\subsection{Estándares generales en las decisiones de contratación}

\subsubsection{Llamado a concurso y proceso de selección inicial}

Los casos analizados muestran que el llamado a concurso se difunde principalmente por tres medios: periódico de difusión nacional, contactos o referencias del cuerpo docentes o del establecimiento, y sitios web institucionales. Los sitios web como medio de difusión son utilizados en menor medida que el resto y se destacan por la posibilidad que tienen de atraer a postulantes jóvenes.

Los procesos de selección comienzan con la revisión de los currículum, los cuales son recibidos en la mayoría de los casos en formato digital (por correo electrónico). Respecto del personal encargado de recibir los currículum e iniciar el proceso de postulación, los cargos más recurrentes que aparecen mencionados son el de director y el del jefe de UTP. En establecimientos privados subvencionados de GSE medio-bajo, la revisión la realizan mayoritariamente directores y jefes de UTP, implicando a un máximo de tres personas en el proceso. En establecimientos de GSE medio-alto, se involucran otros actores (secretarias y psicólogos), lo que llega a sumar hasta siete personas. Por último, en los establecimientos privados pagados de GSE alto, las situaciones son dispares. Hay algunos que tienen un proceso complejo de recepción y procesamiento de solicitudes, en los cuales muchos actores se involucran, y otros en los que todo el proceso recae en manos del psicólogo del establecimiento.

Todos los establecimientos del estudio complementan lo que observan en el CV con al menos una entrevista presencial. Es ahí donde distinguen inicialmente el perfil de los postulantes, y si tienen las habilidades blandas que se esperan de un docente.

Salvo algunos establecimientos subvencionados independientes, la entrevista curricular y la entrevista psicológica también son etapas bastante institucionalizadas y transversales dentro de la postulación. La observación de una clase demostrativa, en cambio, resulta menos común, y se practica principalmente en algunas redes y en establecimientos particulares pagados. De este modo, la observación de una clase es el hito que marca la diferencia entre los procesos de 
contratación de los distintos establecimientos investigados, ya que se incorpora en general en aquellos que tienen mayores recursos y grado de selectividad.

En los establecimientos con más recursos, la mayoría de los directores y jefes de UTP entrevistados mencionan que la selección de profesores en los últimos años se ha transformado en un proceso sistematizado y crecientemente formalizado, con métodos de selección profesionalizados y con un mayor número de etapas. Sin embargo, existen algunos establecimientos subvencionados, tanto independientes como en red, en los cuales el proceso de contratación sigue siendo altamente personalizado e informal.

Los procesos de contratación de profesores varían también en cuanto al nivel de autonomía que tiene el establecimiento. En establecimientos municipales y particulares subvencionados en red, por ejemplo, este procedimiento en general queda fuera del dominio directo de los directores y jefes de UTP. La necesidad se levanta de manera interna, y después la selección es llevada a cabo por la administración central. En esta decisión, los establecimientos en red tienen mayor incidencia, dado que la administración central le entrega una terna de candidatos al director, junto con los informes psicológicos, fortalezas y debilidades, y es sobre la base de esta información que el director toma la decisión. En los establecimientos municipales en cambio, el director solo tiene la capacidad de decidir si el docente seleccionado por la administración se mantiene o no en el cargo.

No obstante, directores de ambos tipos de establecimientos, pueden tener un alto nivel de autonomía en situaciones excepcionales, tales como reemplazos por licencias médicas o contrataciones de apoyo de la Subvención Escolar Preferencial (SEP). En estos casos, los directores pueden proponer o negar candidatos a la autoridad encargada de seleccionar personal, especialmente cuando llevan años en el puesto.

En establecimientos particulares pagados o particulares subvencionados independientes, la selección de profesores es interna 
y autogestionada. Aquí participa una variedad de profesionales del mismo establecimiento: psicólogos, coordinadores académicos, unidad técnica pedagógica y directores. El director, generalmente, es quien se encarga de dar el visto bueno en la última etapa de selección, confiando en el criterio de otros profesionales que llevan a cabo el proceso.

\subsubsection{Filtros y criterios para seleccionar profesores}

Esta sección realiza un desglose de los diversos criterios que mencionan los encargados de contratar profesores.

Uno de los primeros criterios de selección que expresan los cuerpos directivos son las instituciones formadoras en las cuales estudiaron los candidatos a los cargos docentes. Aquí destaca la preferencia por universidades tradicionales, aunque también se le está dando espacio a otras casas de estudio.

Si bien esta es la carta de presentación -sobre todo en establecimientos de nivel socioeconómico alto y medio-, no es definitoria, pues la evaluación suele continuar con la entrevista y la evaluación psicológica.

Paralelamente, existe la postura de que los profesionales egresados de institutos o universidades no acreditadas no se encuentran lo suficientemente preparados. Esto, de modo predominante en establecimientos particulares pagados y subvencionados con nivel socioeconómico medio o alto, en los que provenir de un instituto es razón para descartar un currículum: "Los profesores de instituto vienen muy mal preparados. No saben planificar, no saben hacer una clase, o sea, escriben con falta de ortografía" (directora UTP, particular subvencionado independiente, GSE medio).

Es importante mencionar que si bien es clara la preferencia por universidades acreditadas y con tradición, los establecimientos municipales o de menores ingresos señalan que no siempre pueden descartar por este criterio, debido a que no reciben a postulantes formados en ese tipo de instituciones. 
El segundo criterio que evalúan quienes seleccionan a los docentes es el currículum: cuando el postulante no se dirige al establecimiento por contactos y referencias, este material es el que genera la primera impresión. En el caso de personas jóvenes sin experiencia laboral, se señala que el currículum indica la iniciativa y el grado de involucramiento en actividades extraprogramáticas, como indicador de proactividad y motivación. Para docentes con años de experiencia, se menciona que el currículum determina su estabilidad y permanencia laboral, características que son más valoradas en establecimientos de nivel socioeconómico alto.

Un tercer criterio utilizado es el puntaje PSU, en el caso de profesores con, sin y poca experiencia laboral. Si bien no se trata de un criterio con alta mención dentro de las entrevistas, este es recalcado por un director de un establecimiento subvencionado independiente, quien sostiene que la formación inicial docente no genera ningún tipo de transformación en el futuro profesor, por lo tanto, si obtuvo un bajo puntaje en la PSU tendrá un escaso manejo de la disciplina y didáctica.

Un cuarto criterio que se menciona en varios casos es la concordancia del postulante con el proyecto educativo del establecimiento. En los colegios religiosos es un imperativo tratar de que los elegidos encajen con un perfil determinado. En este sentido, se busca que el docente tenga al menos familiaridad con los ritos religiosos, mientras que a los profesores en jefaturas y cargos de autoridad se les exige tener vínculo con la iglesia.

... que cuando haya una misa sepa cuando hay que pararse y cuando hay que sentarse, que sepa cuándo es el momento de la comunión, cuál es el momento del silencio, cuál es el momento de orar iya? Entonces que tenga una cierta cercanía, no de misa diaria, pero por lo menos que tenga... Que sepa algunos cantos y si no los conoce que con la hojita los pueda cantar, porque es testimonio a los niños (directora, particular subvencionado en red, GSE medio).

$\mathrm{Al}$ igual que en las instituciones religiosas, las escuelas laicas (independiente del tipo de sostenedor o unidad contratadora) también 
buscan un determinado perfil. No obstante, este es menos definido y acotado, aludiendo a valores abstractos o a comportamientos tradicionales.

Otro criterio que aparece en algunos casos es la importancia de los contactos personales, tanto fuera como dentro del establecimiento. Esto, en el momento de elegir entre los postulantes, como también para conseguir personas de confianza que se interesen en la institución y postulen al cargo, lo cual se da principalmente en establecimientos de menores recursos: "uso mis redes internas, entonces voy a donde la profesora que hace... oye, tú, que estás ahí en clases en la universidad, haciendo el postítulo; ¿no hay nadie? ¿compañera tuya o compañero tuyo que pudiera cubrir este tema?" (directora, particular subvencionado en red, GSE medio).

En los establecimientos de nivel socioeconómico medio y alto, y de modo particular los que tienen una formación religiosa, los exalumnos que postulan a cargos docentes presentan ventaja por sobre los demás postulantes.

El sexto criterio utilizado solo en establecimientos educativos vulnerables o con población escolar en riesgo social es la experiencia en colegios de similar vulnerabilidad. En ellos, la tarea de atraer y retener profesores es aún más complicada que en los otros, y por ende, se valoran enormemente a aquellos docentes que "conocen el medio" (directora, particular subvencionado independiente, GSE medio bajo). Los profesores con experiencia en contextos vulnerables son percibidos como profesionales que conocen la realidad de sus estudiantes y comprenden que para ser exitoso en una sala de clases y lograr que los niños aprendan, es necesario aplicar una variedad de técnicas pedagógicas y adaptarse a necesidades diferenciadas.

Los establecimientos pertenecientes a redes educacionales grandes destacan por filtrar a sus postulantes por la formación inicial que poseen: si tienen o no un título universitario, la institución universitaria en la que estudiaron, los puntajes en la prueba Inicia, o si están o no dentro del 50\% superior del ranking de generación. 
En las municipalidades los filtros en general son menores que en las redes grandes. En algunos casos no se aprecian filtros por casa de estudios, en cambio, se realiza un análisis del currículum para ver si el postulante se ajusta al perfil del cargo. En otro caso, el único filtro utilizado es ver si el postulante se encuentra en posesión de su título de docente-universitario o de instituto profesional.

\subsubsection{Dificultad para contratar profesores}

Al momento de tematizar en torno a la dificultad que tienen ciertos establecimientos para contratar profesores se entrelazan dos ejes: la percepción de la baja calidad del profesorado y la desventaja que tienen los contextos vulnerables para retener a los docentes destacados. Es así que el escenario para reclutar a los mejores docentes es altamente competitivo, donde la variable sueldo resulta determinante, dejando en desventaja a la mayoría de los establecimientos de ingresos medios y bajos.

La mayor parte de los entrevistados revela que no tiene la opción de contratar a los mejores docentes, o al menos, a los profesores que ellos desearían en sus aulas. La principal razón argüida, y de forma transversal, es la mala preparación de los docentes: “... tenemos que recibir la herencia de la mala formación de los colegas" (directora, particular subvencionado en red, GSE medio alto). "Lamentablemente se da una alta concentración de no muy buenos profesores. Haciendo un proceso de evaluación de observación de clases, no tengo ningún profesor que yo diga que está en una categoría de competente o destacado" (directora particular subvencionado en red, GSE alto). Este discurso es particularmente frecuente en instituciones con nivel socioeconómico medio para arriba, aunque también aparece en entrevistas en establecimientos de menor ingreso.

En aquellos con menos recursos o prestigio se menciona además que la imposibilidad de contratar a los mejores profesores se da porque estos tienden a ser contratados en colegios de renombre, lo cual se asocia con sueldos más competitivos: "¿Uno tiene la posibilidad de seleccionar a los mejores? No, no tenemos, esos no están aquí. Esos estarán en el Saint George, estarán en el San Ignacio, Las Teresianas, por allá se van" (directora, particular subvencionado en red, GSE 
medio). Aquí, el profesor joven y bueno es el personaje identificado como fácilmente "atrapado por las lucas", que puede partir trabajando por vocación y poco dinero, pero que pronto migra hacia instituciones con mejores condiciones económicas.

La preocupación por los reemplazos y por no dejar a los alumnos sin clases es apremiante en todos los establecimientos, incluyendo a los particulares pagados y municipales de alto rendimiento. Sin embargo, la gran diferencia es que los establecimientos con menos recursos o prestigio se ven frecuentemente obligados a resignarse a elegir entre los pocos profesionales que demuestran interés en el cargo, independientemente de su calidad profesional, lo cual se señala como un motivo de angustia.

Por último, los establecimientos -a excepción de aquellos de alto GSE-, deben enfrentarse al problema de convencer a los docentes seleccionados para quedarse. Esto debido a diversos testimonios acerca de personas que rechazan el cargo al enterarse del sueldo y las condiciones laborales, al igual que anécdotas de profesores que aceptan el cargo y luego no llegan.

\subsubsection{Rotación de docentes}

La mayoría de los establecimientos estudiados valora la estabilidad de las plantas profesionales en la medida en que beneficia la calidad del proyecto educativo. En contextos de baja rotación, los cupos disponibles son fruto de la jubilación, renuncias (causadas por cambio de domicilio o mejores oportunidades), despidos o cambios administrativos del colegio. Esto es más común en los establecimientos municipales, influenciados por el marco legal del Estatuto Docente.

Por el contrario, en contextos de alta rotación, los cuerpos directivos se ven obligados a abrir procesos de selección de docentes, incluso más de una vez al año. Una de las principales razones por las que surge la necesidad de buscar nuevos docentes son las licencias médicas o bajas laborales por enfermedad. Esto exige encontrar reemplazos en un período corto, obligando a la integración temporal de personas menos experimentadas a la comunidad escolar, siendo 
274 PERCEPCIONES ACERCA DEL PROCESO DE BÚSQUEDA Y CONTRATACIÓN DE DOCENTES EN CHILE: NUDOS CRÍTICOS E INEQUIDAD DEL SISTEMA - M. Ortúzar, P. Ayala, C. Flores, C. Milesi

esta una realidad más frecuente en establecimientos que se insertan en contextos más vulnerables:

(...) en el colegio más vulnerable dentro de la fundación, la experiencia es muy dura porque ahí, en mayo uno ya sabía que había nueve o diez profesores con licencia psiquiátrica. Es que es muy duro el ambiente, muy duro, muy duro. Entonces, además de la rotación de profesores, ahí se iba la reemplazante de la reemplazante (directora, particular subvencionado en red, GSE medio).

Una segunda razón que explica la alta rotación es la denominada "fuga de talentos" hacia establecimientos de mayor nivel socioeconómico. "Hay un grupo de profesores que nos dejan, sobre todo los que en la evaluación docente les va bien, son destacados y son competentes, buscan otros colegios en que les pagan mejor" (director, municipal DAEM, GSE bajo). "Un profesor bien catalogado rápidamente es capturado, las manos negras, las grúas, se hacen cargo" (directora, particular subvencionado en red, GSE medio). De manera transversal, los encargados de contratar a profesores en diferentes tipos de establecimientos creen que la fuga de talentos es motivada casi en un $100 \%$ por razones salariales. No obstante, en instituciones particularmente vulnerables, la rotación de docentes, sean de excelencia o no, también puede ser gatillada por una realidad laboral compleja y hostil:

O sea un profesor que tuvimos duró un día, no aguantó al curso y se fue. Por eso yo también me fijo en que si ha trabajado en otros establecimientos y de qué tipo, si ha trabajado en un colegio vulnerable y duró harto tiempo es porque sabe del manejo de curso y eso es lo más importante (jefa UTP, particular subvencionado independiente, GSE medio).

\subsubsection{Cuidados y estrategias para retener profesores}

Una práctica común para facilitar la inserción de los docentes son los ritos o actividades de introducción para profesores nuevos dentro de la institución. En la mayoría de los establecimientos estudiados, se les exige a los profesores recién contratados que se integren antes del comienzo del año escolar, para así aprender respecto de la misión de la institución y de su forma de operar. En estas instancias se tratan 
principalmente temas relacionados con el proyecto educativo, la visión corporativa, el organigrama de la institución y las funciones administrativas.

Otra práctica común corresponde a los procesos de inducción y acompañamiento para los profesores nuevos, los cuales buscan orientar y acoger al nuevo docente, para así facilitar su integración a la comunidad escolar. Estos procesos funcionan de manera más formal en establecimientos particulares pagados y redes educacionales, así como en instituciones de nivel socioeconómico medio y alto, en estos casos cuando son docentes nóveles la inducción se enfoca en entregar contenidos pedagógicos y de manejo de aula. Algunas de las actividades que incluyen son las mentorías, observación de aula, evaluaciones calendarizadas y talleres de desarrollo profesional.

Junto con los procesos de inducción formales mencionados, en los establecimientos se pueden dar procesos informales, que surgen como resultado de dinámicas positivas dentro de la institución. Este es el caso de los lazos de ayuda que se generan de modo natural entre los profesores y el apoyo de la comunidad hacia los nuevos docentes, donde "todos se transforman en una especie de profesor guía" del profesional que se integra y "les dan algunos tips" sobre cómo tratar a los alumnos" (jefa UTP, particular subvencionado en red, GSE medio bajo).

El sueldo es también una estrategia percibida como crítica para retener a los profesores. En algunos establecimientos se negocia y aumenta el salario de los profesores más destacados, con el fin de evitar su fuga. Esta estrategia se encuentra directamente vinculada con el nivel socioeconómico del establecimiento y su disponibilidad de recursos.

En aquellas instituciones donde no es factible un bono o aumento de sueldo, la estrategia que se menciona para retener a los profesores es aludir a la existencia de un grato ambiente laboral, trabajar en un ambiente relajado, de reconocimiento, donde se puede aprender y trabajar en forma segura. 
276 PERCEPCIONES ACERCA DEL PROCESO DE BÚSQUEDA Y CONTRATACIÓN DE DOCENTES EN CHILE: NUDOS CRÍTICOS E INEQUIDAD DEL SISTEMA - M. Ortúzar, P. Ayala, C. Flores, C. Milesi

\subsection{El caso de los profesores recién egresados}

\subsubsection{Prácticas profesionales y profesores no titulados}

Un tema destacado en el caso de los profesores jóvenes son las prácticas profesionales y su función como proceso de atracción de futuros docentes. Desde la perspectiva de los administradores de los establecimientos las prácticas son, entre otras cosas, una instancia para compartir el proyecto educativo e interesar a los profesores nóveles en un ambiente laboral que de otra forma no hubiesen considerado. Los reemplazos funcionan de manera similar, ya que algunos establecimientos toman este corto período como proceso de introducción, y luego los contratan de forma definitiva.

En esta línea, contratar a profesores que aún no están titulados, resulta una alternativa ante un escenario de escasez -por ejemplo, para atraer profesores de Física o Matemática-. Esta es una estrategia utilizada en mayor medida en establecimientos subvencionados independientes y en establecimientos de nivel socioeconómico bajo.

\subsubsection{Lo mejor de los profesores recién egresados}

En general, independientemente de la visión negativa que existe frente a la formación inicial docente, los directores y jefes de UTP tienen una preferencia marcada por los profesores recién egresados. Un primer motivo tiene que ver con la vocación, las habilidades blandas y el trato que los docentes jóvenes tienen hacia los niños, más allá de su nivel de conocimientos profesionales. Un segundo motivo dice relación con las ganas y la facilidad para aprender. En este sentido, se entiende al docente joven como un profesional moldeable al proyecto educativo en el que se inserta, por lo que ante una buena disposición, la falta de experiencia no es considerada como un inconveniente, sino como un potencial; en contraposición con el profesor con más años de experiencia y con una mayor resistencia frente al cambio. Un tercer motivo dice relación con los contenidos, pues los profesores jóvenes pueden llegar con nuevos métodos o tecnologías educativas. Este discurso aparece principalmente en establecimientos de nivel socioeconómico bajo. Por último, una cuarta razón que explica por qué optan por contratar a profesores con menos experiencia, es que ellos suelen aceptar sueldos más bajos. Esta motivación se 
menciona también particularmente en establecimientos de menor nivel socioeconómico.

\subsubsection{Cuidados y estrategias para retener a los profesores jóvenes}

Por lo general, los profesores jóvenes reciben una inducción, se les acompaña, capacita y evalúa de forma reiterada, para así contrarrestar su poca experiencia en la docencia. Adicionalmente, salvo algunas excepciones, se les reserva la jefatura de curso para cuando tengan más tiempo de experiencia.

\subsubsection{Evaluación de los principales problemas de los profesores} recién egresados

El problema más comúnmente encontrado entre los profesores jóvenes es el deficiente manejo de grupo y los problemas de autoridad ante los alumnos. De modo particular, las relaciones excesivamente horizontales que establecen los profesores jóvenes con sus alumnos es visto como un problema que interfiere en el proceso de aprendizaje. Esto se presenta de manera transversal en los distintos tipos de establecimientos, aunque con mayor énfasis en aquellos que se insertan en contextos de menor nivel socioeconómico, donde al poco manejo de aula del profesor se le suelen agregar otros problemas relacionados con desafíos provenientes de los hogares de los estudiantes.

Asimismo, en los docentes jóvenes se observa poco manejo de la diversidad que existe realmente en los establecimientos. En general se apunta al problema de tener una formación excesivamente teórica y orientada a un alumno determinado, cuando la realidad de la sala de clases es otra.

La planificación, el manejo de los tiempos y saber estructurar el contenido de acuerdo con los imprevistos que se generan en una hora de clases son deficiencias comunes que suelen relacionarse con la falta de familiaridad de las dinámicas que se dan dentro de la sala de clases.

Asimismo, algunos entrevistados mencionan problemas de tipo administrativo, como el ausentismo e irresponsabilidad: "Si les duele 
el pelo se quedan en la casa" (directora, particular subvencionado en red, GSE medio); "Agarran el teléfono y te dicen joye, sabes que no voy a ir!” (jefe UTP, municipal corporación, GSE bajo), lo que es menos frecuente en profesores mayores o con más experiencia.

Por último, otra rama de problemas en la evaluación de los profesores jóvenes tiene que ver con la convivencia con los docentes más antiguos. Se dificulta un trabajo colaborativo dentro del colegio cuando existen roces entre las distintas generaciones de profesores, particularmente cuando los profesores con más experiencia se ven amenazados por la figura del profesor joven y bien evaluado. Por otro lado, los jóvenes "moldeables" y "dóciles" de los que hablan los entrevistados, también pueden aprender las malas prácticas de los profesores con mayor antigüedad, generándose un proceso de aprendizaje poco virtuoso. En resumidas cuentas, "el profesor también se adecúa a la realidad, y se malea de acuerdo a la realidad" (directora UTP, particular subvencionado en red, GSE alto).

\section{Conclusiones}

A continuación se presentan las principales conclusiones del estudio para luego discutir sus implicancias para las políticas públicas.

En general se observa que los procesos de búsqueda y contratación son muy variados. Estos van desde procesos simples e informales, donde un número reducido de actores revisa los currículum y realiza entrevistas para dilucidar entre un par de postulantes, a procesos más complejos que involucran a otros profesionales que revisan currículum, entrevistan y observan clases. Generalmente los establecimientos en red son los que presentan procesos más complejos, seguidos por los colegios privados pagados. El caso de los municipios es variable, pero en general se da que establecimientos con mayores recursos realizan una gestión más compleja en el proceso de contratación.

Dentro de este proceso existen ciertos fenómenos identificados como nudos críticos por parte de los directores y jefes de UTP. Entre estos destacan la autonomía de los directores, la rotación 
de profesores, las etapas que diferencian a los distintos tipos de establecimientos, los criterios para seleccionar a los profesores y la dificultad para contratar docentes.

Respecto de la autonomía de directores, en establecimientos municipales y pertenecientes a redes, el proceso y la elección generalmente se hacen a nivel central, con la diferencia de que las redes suelen darles mayor capacidad de elección a los directores. Otra diferencia entre las redes de colegios y los establecimientos municipales es que en las primeras tiende a existir mayor grado de selectividad y de recepción de profesores jóvenes. Si bien, en teoría, los establecimientos municipales no tienen mayor capacidad de participar en los procesos de selección llevados a cabo por la administración central, en la práctica existe un diálogo en el cual el director del establecimiento puede proponer o negar candidatos. Se observa que en aquellos establecimientos municipales que han desarrollado una mayor capacidad de gestión, los directores tienen mayor autonomía en el proceso de contratación de profesores.

En cuanto a las etapas que conforman el proceso de selección, todos los establecimientos del estudio, incluso aquellos con menores recursos, complementan lo que observan en el currículum con, al menos, una entrevista presencial. Es ahí donde se distingue inicialmente el perfil del postulante y sus habilidades blandas. Salvo algunos establecimientos subvencionados independientes, la entrevista psicológica también es una etapa bastante institucionalizada y transversal dentro de la postulación para un cargo docente. La observación de una clase demostrativa, en cambio, es menos común, y viene a ser el hito que marca la diferencia entre los procesos de contratación de los distintos establecimientos investigados, ya que esta se contempla en los colegios que tienen mayores recursos y grado de selectividad.

Un tercer nudo crítico son los criterios para seleccionar a los profesores. En general existe una preferencia por egresados de instituciones tradicionales, selectivas y de mayor trayectoria, así como también se intenta evitar contratar a egresados de institutos profesionales o egresados de universidades no acreditadas. Asimismo, 
se observa como criterio de selección, transversal a los distintos tipos de establecimientos y niveles socioeconómicos, la "afinidad sociocultural" del docente con el establecimiento, en términos de la experiencia educativa o laboral previa, grupo socioeconómico de pertenencia, o afiliación religiosa. En este mismo sentido, en contextos vulnerables se destaca la importancia de que el docente tenga experiencia de trabajo en contextos similares, entendiendo esto como un indicador de si el candidato es un "conocedor del medio".

La rotación de profesores constituye otro nudo crítico dentro de este proceso. En general, los establecimientos valoran la estabilidad de la planta docente, por lo que intentan mantener baja la tasa de rotación. Esto conlleva a que los cupos para nuevos profesores se abran principalmente por dos razones: licencias médicas y fuga de talentos. La primera, genera una necesidad inmediata de encontrar un reemplazo en poco tiempo, mientras que la segunda es un problema constante para establecimientos de nivel socioeconómico bajo, medio y, en menor medida, medio alto, que perciben que no pueden competir con los sueldos de los establecimientos de nivel socioeconómico alto.

Por último, un nudo especialmente complejo en el proceso es la dificultad general para atraer y contratar profesores, sobre todo aquellos considerados como "buenos". De acuerdo con los que contratan, los establecimientos particulares pagados logran atraer a los mejores profesores principalmente gracias a sus altos salarios.

Este trabajo también se propuso explorar la evaluación que hacen los supervisores respecto de las fortalezas y debilidades en el desempeño de los profesores recién contratados con poca o nula experiencia previa.

La evidencia refleja que en general existe una alta valoración por los profesores recién egresados, ya sea por la vocación, las habilidades blandas, el trato hacia los niños, la facilidad para aprender, la apertura a ser corregidos y la adaptabilidad al proyecto educativo, junto con la posibilidad de que incorporen nuevos métodos y tecnologías educativas. Los directivos de establecimientos pertenecientes a GSE bajo agregan que estos suelen aceptar sueldos más bajos. 
Sin embargo, también se perciben ciertas debilidades en los profesores recién egresados, como la falta de preparación, y el mal manejo de contenidos y de la normativa vigente. La falencia más mencionada y transversal, es el deficiente manejo de grupo y los problemas de autoridad que tienen los profesores jóvenes frente a sus alumnos, lo cual es percibido en mayor medida en los contextos de menor nivel socioeconómico, donde al poco manejo de grupo del profesor se agregan problemas adicionales asociados a la vulnerabilidad de los alumnos. Se percibe que la falta de preparación para el manejo de la diversidad dentro del aula y las dificultades en la planificación de la clase son fruto de una formación inicial excesivamente teórica y orientada a un tipo de alumno determinado. Por otra parte, se detectan problemas ligados al ausentismo y de escaso trabajo colaborativo con los profesores con más experiencia.

Respecto de las estrategias para retener profesores, en especial a los recién egresados, los entrevistados destacan las estrategias de introducción y procesos de inducción, aumentos salariales y cuidados laborales especiales. Los procesos de introducción buscan integrar al nuevo docente, dando a conocer el proyecto educativo y cuestiones operativas. La inducción, por su parte, es el proceso de acompañamiento a los profesores recién egresados en el aula, lo cual se realiza de modo más formal en establecimientos particulares pagados y redes educacionales, así como en instituciones de nivel socioeconómico medio y alto. Respecto de aumentos salariales, existen establecimientos donde el profesor destacado puede entrar a negociar o bien recibir un bono. Lo anterior es una práctica de rutina únicamente en establecimientos particulares pagados, siendo excepcional en otros. Por último, en cuanto a cuidados específicos para profesores recién egresados, algunos establecimientos ofrecen mayor nivel de acompañamiento y, dentro de lo posible, esperar un tiempo para otorgarle una jefatura.

\section{Recomendaciones para la política pública}

Es importante destacar que los esfuerzos recientes que se han hecho en Chile por atraer buenos candidatos a la docencia -a través de iniciativas públicas como la ley de Carrera Docente, Beca Vocación 
de Profesor y Elige Educar-, tienden a generar incentivos para los profesores, más que para los contratadores, además de no estar suficientemente orientadas a dar incentivos para que profesores calificados se desempeñen en contextos vulnerables. De acuerdo con los resultados de este estudio, se sugiere explorar la posibilidad de complementar estas políticas con otras, orientadas a fortalecer los procesos de reclutamiento de profesores, que apoyen a los establecimientos educacionales, sobre todo a los más vulnerables, a potenciar buenas prácticas en el proceso de reclutamiento, selección, contratación y retención de docentes. A continuación se mencionan algunas posibles líneas de políticas en esta área.

Una de ellas es otorgar apoyos en gestión, bonos o incentivos monetarios con el objetivo de potenciar las capacidades de los establecimientos en la fase inicial de reclutamiento de profesores, una vez que se generan vacantes. Por ejemplo, el estudio provee evidencia respecto de la importancia de las prácticas profesionales y alianzas con instituciones de formación inicial, como una posible forma de atraer a buenos postulantes, práctica que puede ser potenciada dentro de los establecimientos. Este apoyo inicial podría diversificar y elevar la calidad de postulantes que tiene cada uno para seleccionar a sus futuros profesores. Al respecto, estudios internacionales muestran que los establecimientos que generan lazos formales con instituciones de formación que comparten visiones religiosas o filosóficas son más exitosos para atraer y reclutar docentes (Egalite, Jensen, Stewart \& Wolfet, 2014).

Una segunda línea de políticas estaría enfocada en etapas posteriores del proceso, como lo son los procesos de selección y evaluación de los postulantes. Stronge y Hindman (2003) plantean que en un escenario ideal, el proceso de selección debiera incluir elementos que permitan la observación del desempeño. Sin embargo, esta es una práctica que debido a las restricciones presupuestarias y de tiempo, como se sugiere en este estudio, podría no estar realizándose a nivel masivo en el país. A pesar de su alto costo, la herramienta de observación de clases es muy relevante en el contexto chileno, el que se caracteriza por la baja selectividad de las instituciones formadoras de docentes y en el que la percepción de calidad docente 
de los encargados de contratación en los establecimientos parece ser baja. Es así como para los establecimientos de mayor vulnerabilidad, que son los que reciben la mayor proporción de candidatos con credenciales menos valoradas, sería altamente beneficioso contar con apoyo de recursos para poder evaluar en el aula el desempeño de sus postulantes. Apoyos en gestión por parte del Ministerio de Educación y recursos de la Ley SEP se podrían utilizar para estos efectos.

Un factor que aparece como relevante en el estudio es el poco manejo de grupo y de la diversidad de la sala de clases de los profesores recién egresados y las dificultades adicionales que esto implica cuando se trabaja en contextos más vulnerables. Una estrategia posible en esta área es apoyar a los profesores principiantes en este aspecto a través de programas de inducción que entreguen acompañamiento y estrategias para trabajar con contextos de alta vulnerabilidad, además del apoyo pedagógico y de contenidos que tienen los programas de inducción tradicionales. De este modo, los procesos de inducción con foco adicional en contextos vulnerables incorporarían contenidos específicos relacionados con el manejo de grupo y de la diversidad dentro de la sala de clases, de forma de enfrentar de mejor forma la realidad en este tipo de establecimientos. Esto podría ser incorporado al componente de inducción de la Ley de Carrera Docente, el que se encuentra actualmente en proceso de implementación por parte del Mineduc. Otra estrategia posible es enfatizar, en las instituciones formadoras de docentes, la formación teórica y práctica de los futuros docentes respecto de lo que implica trabajar en contextos diversos y vulnerables. Para implementar ambas estrategias se hace necesario fortalecer el vínculo entre establecimientos e instituciones formadoras de docentes, ya sea para que el proceso de inducción se focalice de manera explícita en contenidos relevantes para contextos vulnerables, como para que la formación inicial prepare de mejor manera a los docentes para enfrentarse a enseñar en dichos contextos. 
284 PERCEPCIONES ACERCA DEL PROCESO DE BÚSQUEDA Y CONTRATACIÓN DE DOCENTES EN CHILE: NUDOS CRÍTICOS E INEQUIDAD DEL SISTEMA - M. Ortúzar, P. Ayala, C. Flores, C. Milesi

\section{Referencias}

Ávalos, B. (2014). La formación inicial docente en Chile: tensiones entre políticas de apoyo y control. Revista estudios pedagógicos, 40, 11-28. https://dx.doi.org/10.4067/S0718-07052014000200002

Akiba, M., LeTendre, G. K., \& Scribner, J. P. (2007). Teacher quality, opportunity gap, and national achievement in 46 countries. Educational Researcher, 7(36), 369-387. http://doi.org/10.3102/0013189X07308739

Ballou, D. \& Podgursky, M. (1998). Teacher recruitment and retention in public and private schools. Journal of Policy Analysis and Management, 3(17), 393-417. http://dx.doi.org/10.1002/(sici)15206688(199822)17:3<393::aid-pam2>3.3.co;2-9

Balter, D. \& Ducombe, W. (2006). Staffing classrooms: Do teacher hiring practices affect teacher qualifications? Recuperado de https://www.maxwell.syr. edu/uploadedFiles/cpr/research/cpr_research_education_finance_ policy/aefa2006.pdf

Balter, D. \& Duncombe, W. (2005). Teacher hiring practices in New York state school districts (Informe preparado para el Education Finance Research Consortium). Recuperado de https://www.maxwell.syr.edu/ uploadedFiles/cpr/people/faculty/duncombe/exec-summary(1).pdf

Barber, M. \& Mourshed, M. (2007). Cómo hicieron los sistemas educativos con mejor desempeño del mundo para alcanzar sus objetivos (Informe para el Programa de Promoción de la Reforma Educativa en América Latina y El Caribe, PREAL). Recuperado de www.oei.es/historico/pdfs/ documento_preal41.pdf

Béteille, T. \& Loeb, S. (2009). Teacher quality and teacher labor markets. En G. Sykes, B. Schneider \& D. N. Plank (Eds.), Handbook of education policy research (pp. 596-612). New York: Routledge.

Boyd, D., Lankford, H., Goldhaber, D., \& Wyckoff, J. (2007). The effect of certification and preparation on teacher quality. The Future of Children, 1(17), 45-68. http://dx.doi.org/10.1353/foc.2007.0000

Boyd, D., Lankford, H., Loeb, S., \& Wyckoff, J. (2013). Analyzing the determinants of the matching of public school teachers to jobs: Disentangling the preferences of teachers and employers. Journal of Labor Economics, 31(1), 83-117. http://dx.doi.org/10.1086/666725

Cabezas, V., Gallego, F., Santelices, V., y Zarhi, M. (2011). Factores correlacionados con las trayectorias laborales de docentes en Chile, con especial énfasis en sus atributos académicos (Proyecto Fonide $N^{\circ}$ FS511082-2010). Santiago de Chile: Ministerio de Educación. 
Centro de Investigación Avanzada en Educación, CIAE. (2014). El 40\% de los profesores nuevos abandona la profesión al quinto año. Recuperado de http://www.ciae.uchile.cl/index.php?page=view_noticias\&id=46 osrexterno=boletin

Cooper, J. \& Alvarado, A. (2006). Preparation, recruitment, and retention of teachers. Recuperado de http://www.unesco.org/iiep/PDF/Edpol5.pdf

Corvalán, J., Elacqua, G., y Salazar, F. (2009). El sector particular subvencionado en Chile. Tipologización y perspectivas frente a las nuevas regulaciones (Proyecto Fonide $N^{\circ}$ 69). Santiago de Chile: Ministerio de Educación.

Darling-Hammond, L. (2003). Keeping good teachers. Choice Reviews Online, 42, 42-1077. http://dx.doi.org/10.5860/choice.42-1077

Egalite, A., Jensen, L., Stewart, T., \& Wolf, P. (2014). Finding the right fit: Recruiting and retaining teachers in Milwaukee choice schools. Journal of School Choice, 1(8), 113-140. http://dx.doi.org/10.1080/15582159 .2014 .875418

Futernick, K. (2007). A possible dream: Retaining California's teachers so all students learn. Sacramento: California State University. Recuperado de https://www.calstate.edu/teacherquality/documents/possible_dream. pdf

Gaete, A., Castro, L., Pino, F., y Mansilla, D. (2016). Abandono de la profesión docente en Chile: motivos para irse del aula y condiciones para volver. Trabajo no publicado. Recuperado de https://www.researchgate. net/profile/Alfredo_Gaete/publication/306305947_Abandono_de_ la_profesion_docente_en_Chile_Motivos_para_irse_del_aula_y_ condiciones_para_volver/links/57b7611908aedfe0ec938969.pdf

Glaser, B. \& Strauss, A. (1967). The discovery of grounded theory. Chicago, IL: Aldine Publishing.

Grau, F., Maturana, C., Paterson, M., y Valenzuela, C. (2012). Cómo los establecimientos educacionales escogen a sus profesores: estudio de difusión, selección y contratación de docentes en la Región Metropolitana. Santiago de Chile: Ingeniería UC Propone.

Guarino, C., Santibáñez, L., Daley, G., \& Brewer, G. (2004). A review of the research literature on teacher recruitment and retention. Review of Education Research, 2(76), 173-208.

Hanushek, E. \& Rivkin, S. (2007). Pay, working conditions, and teacher quality. The future of children, 1(17), 69-86. http://dx.doi.org/10.1353/ foc. 2007.0002 
Harris, D., Rutledge, S., Ingle, W., \& Thompson, C. (2010). Mix and match: What principals really look for when hiring teachers. American Education Finance Association, 2(5), 228-246. http://dx.doi.org/10.1162/ edfp.2010.5.2.5205

Haycock, K. \& Peske, B. H. G. (2006). Teaching inequality: How poor and minority students are shortchanged on teacher quality. Recuperado de https://edtrust.org/resource/teaching-inequality-how-poor-andminority-students-are-shortchanged-on-teacher-quality/

Ingersoll, R. (2001). Teacher turnover and teacher shortages: An organizational analysis. Recuperado de http://repository.upenn.edu/gse_pubs/94.

Johnson, S. M. (2006). The workplace matters: Teacher quality, retention and effectiveness. Washington DC: National Education Association.

Ladd, H. (2011). Teachers' perceptions of their working conditions: How predictive of planned and actual teacher movement? Educational Evaluation and Policy Analysis, 2(33), 235-261. http://dx.doi. org/10.3102/0162373711398128

Leithwood, K., Seashore Louis, K., Anderson, S., \&Wahlstrom, K. (2004). How leadership influences student learning. Learning from Leadership Project. Recuperado de http://www.wallacefoundation.org/knowledge-center/ Documents/How-Leadership-Influences-Student-Learning.pdf

Liu, E. (abril, 2005). Hiring, job satisfaction, and the fit between new teachers and their schools. Trabajo presentado en la American Educational Research Association. Montreal. Recuperado de http://projectngt.gse. harvard.edu/files/gse-projectngt/files/liu_aera_2005_hiring_and_job_ satisfaction.pdf

Loeb, S., Darling-Hammond, L., \& Luczak, J. (2005). How teaching conditions predict teacher turnover in California schools. Peabody Journal of Education, 3(80), 44-70. http://dx.doi.org/10.1207/ s15327930pje8003_4

Loeb, S. \& Reininger, M. (2004). Public policy and teacher labor markets. What we know and why it matters. Recuperado de http://cepa.stanford.edu/ content/public-policy-and-teacher-labor-markets-what-we-know-andwhy-it-matters

Maxwell, J. A. (2013). Qualitative research design: An interactive approach. Thousand Oaks, CA: Sage.

Ortúzar, M., Flores, C., Milesi, C., y Cox, C. (2009). Aspectos de la formación inicial de docentes y su influencia en el rendimiento académico de los alumnos. Santiago: UC, Centro de Políticas Públicas. 
Paredes, R., Bogolasky, F., Cabezas, V., Rivero, R., y Zahri, M. (2013). Los determinantes del primer trabajo para profesores de educación básica en la Región Metropolitana. (Proyecto Fonide No: F611105). Santiago de Chile: Ministerio de Educación.

Rivero, R. (2015). The link of teacher career paths on the distribution of high qualified teachers: A Chilean case study. Archivos Analíticos de Políticas Educativas, 23(73), 1-35. http://dx.doi.org/10.14507/epaa.v23.1710

Ruffinelli, A. y Guerrero, A. (2009). Círculo de segmentación del sistema educativo chileno. Calidad en la Educación, 31, 20-44.

Ruffinelli, A. (2013). La calidad de la formación inicial docente en Chile: la perspectiva de los profesores principiantes. Calidad en la educación, 39, 117-154. https://dx.doi.org/10.4067/S0718-45652013000200005

Sanders, W. \& Rivers, J. (1996). Research project report: Cumulative and residual effects of teachers on future student academic achievement. Knoxville, TN: University of Tennessee Value-Added Research and Assessment Center.

Stake, R. (2005). Qualitative case studies. En N. Denzin \& Y. Lincoln (Eds.), The Sage Handbook of Qualitative Research (pp. 443-466). Thousand Oaks, CA: Sage Publications Ltd.

Stake, R. (1995). The art of case study research. California: Sage.

Stronge, J. \& Hindman, J. (2003). Hiring the best teachers. Educational Leadership, 8(60), 48-52.

Toledo, G. y Valenzuela, J. P. (2012). Ordenamiento de profesores y estudiantes entre y dentro de los establecimientos escolares: el caso de Chile. Recuperado de http://www.econ.uchile.cl/uploads/publicacion/b77345cb20543ea 1c590a3b4985fc7fc2214dbfd.pdf

Wang, H. \& Fwu, B. (2013). "Once hired, seldom gone": The deliberation process of beginning teachers in Taiwan in deciding to stay in teaching. Teaching and Teacher Education, 37, 108-118. http://dx.doi. org/10.1016/j.tate.2013.10.004

Weiss, E. M. (1999). Perceived workplace conditions and first-year teachers' morale, career choice commitment, \& planned retention: A secondary analysis. Teaching and Teacher Education, 8(15), 861-879. http://dx.doi. org/10.1016/s0742-051x(99)00040-2

Recibido: 31/08/2015

Aceptado: 15/09/2016 